\title{
Summary of the Eleventh Annual University of Wisconsin Land O'Lakes Bioanalytical Conference
}

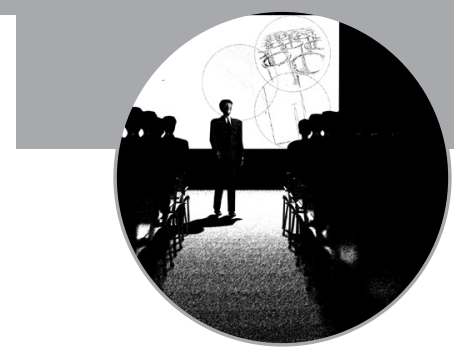

\author{
Eleventh Annual University of Wisconsin Land O'Lakes Bioanalytical Conference \\ University of Wisconsin, WI, USA, 19-23 July 2010
}

This University of Wisconsin School of Pharmacy Bioanalytical Conference is arranged by the Extension Services in Pharmacy, the professional development department within the School. The purpose of this 4 day conference is to provide an educational forum to discuss issues and applications associated with the analysis of xenobiotics, metabolites, biologics and biomarkers in biological matrices. The conference is designed to include and encourage an open exchange of scientific and methodological applications for bioanalysis. To increase the interactive nature of the conference, the program was a mixture of lectures, poster sessions, round-table discussions and workshops. This paper summarizes the presentations at the Eleventh Annual Conference.

Keywords: biologics $\approx$ electronic notebooks $\approx$ laboratory frustrations $\approx$ method development $\approx$ method validation $\approx$ new technologies - professional development $=$ tissue sample analysis

The Eleventh Annual Land O'Lakes Bioanalytical Conference, titled 'A new decade of bioanalysis of large and small molecules', was held on 19-23 July 2010 at Devil's Head Resort in Merrimac, WI, USA. This conference brings together an international group of bioanalytical scientists with the express intent of discussing cutting-edge science for small molecule and biologic bioanalysis in a relaxed atmosphere that also promotes networking opportunities. The conference provides an educational forum with presentations by scientific leaders from academia, industry, contract laboratories and regulatory authorities.

The success of the program is due to the work of the planning committee, which consists of scientists involved with bioanalysis on a daily basis and their ability to identify timely topics, and presenters who are experts in the field. Specific objectives for the conference and a list of the planning committee members can be found on the University's website [101].

\section{Four plenary sessions}

Morning meetings consisted of four plenary sessions focusing on specific themes associated with bioanalytical issues. The first plenary session, opened and moderated by the Conference Chair, Michael Hayes (Novartis), focused on new technologies with applications in bioanalysis. David Clemmer (Indiana University, IN, USA) started the conference with a presentation on new developments in ion mobility MS
(IMMS) techniques. He used polyethylene glycol (PEG), a linear molecule, to illustrate the capability of IMMS to measure molecular shape and used bradykinin (a peptide of nine amino acids) to demonstrate the capability of IMMS in study conformation change. He also spoke about new developments that provide a dramatic increase in the resolving power of ion mobility-based separations. There is a growing need for analysis of complex biological mixtures (e.g., high-molecular-weight drugs and biomarkers) and ion mobility has significant potential in these areas. The presentation showed how ion mobility can reduce chemical background noise and provide structural information. New instrument geometries have been shown to provide even greater resolution and have the potential to provide powerful new tools for analysis. Robert Plumb (Waters Corporation) presented developments in chromatography. The dominance of MS as the method of detection has allowed considerable simplification of sample preparation. Developments in HPLC have led to very short run times, however, the manner in which these have been applied often results in reduced selectivity, which creates challenges for potential interference from matrix and metabolites. UHPLC provides a significant increase in peak capacity that can provide very fast, high-sensitivity separations, or can be utilized for high-efficiency separations for applications such as metabolite analysis. Capillary-scale LC and microfluidic devices have the potential to
James E DeMuth ${ }^{\dagger 1}$, Michael J Hayes ${ }^{2}$, Lakshmi Amaravadi, Mark E Arnold4, Chad Briscoe ${ }^{5}$, Robert P Clement ${ }^{6}$, Stacy $\mathrm{Ho}^{7}$, Qin $\mathrm{C} \mathrm{Ji}^{4}$ \& Douglas J Turk ${ }^{8}$

'University of Wisconsin, 777 Highland Ave, Madison, WI 53705, USA ${ }^{2}$ Novartis Pharmaceuticals Corporation, East Hanover, NJ, USA ${ }^{3}$ Biogen Idec, Cambridge, MA, USA ${ }^{4}$ Bristol-Myers Squibb Company, Princeton, NJ, USA ${ }^{5}$ PRA International, Lenexa, KS, USA ${ }^{6}$ Merck Research Laboratories, Summit, NJ, USA ${ }^{7}$ Genzyme Corporation, Waltham, MA, USA

${ }^{8}$ NoAb BioDiscoveries Inc., Mississauga, Ontario, Canada ${ }^{\dagger}$ Author for correspondence: Tel.: + I 6082622422 Fax: +| 608262243 |

E-mail: jedemuth@pharmacy.wisc.edu 
provide high sensitivities from small samples such as blood spots; however, they present challenges for instrument-scanning speed. Paul Grimsrud (University of Wisconsin-Madison, WI, USA) discussed innovations in the study of proteins and their post-translational modifications. New instrument approaches, such as electron-transfer dissociation, provide new tools for the study of proteins that complement traditional methods. Greater availability of high-resolution MS instruments in a variety of configurations has aided the study of proteins. Advances in sample preparation, combined with methods such as isobaric tagging derivatives, have also contributed to the study of protein systems. Combining these new techniques allows advances in areas such as the study of phosphorylation of proteins. Gary J Van Berkel (Oak Ridge National Laboratory, TN, USA) presented on recent developments in MS-based methods of surface analysis for drug imaging and quantitation. These methods are applicable to a variety of sample types, such as dried blood spot analysis, high-throughput analysis of dried samples by diversity array technology and laser diode thermal desorption, as well as imaging of thin tissue slices with liquid microjunction surface sampling. These approaches have resulted in a significant improvement in accurate and sensitive quantitative measurements from a variety of solid surfaces.

Wednesday's plenary session addressed some of the current analytical challenges related to biologics and provided new approaches to address these challenges. Ago Ahene (Pharmargo LLC) introduced the topic of free drug and total drug measurements using either 'specific' or 'generic' assay formats for monoclonal antibodies (mAbs). Utility of measuring 'free' versus 'total' drug in preclinical and clinical development stage programs and various ligand-binding assay formats that can be utilized to measure these analytes were discussed. Four different biologic case study examples were presented to illustrate that free versus total drug measurements may or may not provide comparable data with respect to exposure, half-life and clearance, and that appropriate analytes to be measured should be selected to fit the purpose. Ola Saad (Genentech) discussed bioanalytical challenges associated with measurement of antibody drug conjugates (ADCs) and highlighted the utility of new platforms for such applications. After an overview of ADCs in clinical development, their typical structure, and the utility of a combination of immunoassaybased plus LC-MS/MS-based approaches, she presented a trastuzuamb-DM1 case study. The major analytes of interest were defined as 'active drug', 'total antibody' and 'free drug DM1'. Structures present in plasma and sample processing methods for measurement of the three species of DM1 were discussed, along with novel analytical approaches to measurement of intact ADCs using affinity-capture MS and automation possibilities for such methods. Frank-Peter Theil (Genentech) changed the focus slightly to address the latest challenge related to drug-drug interactions (DDIs) between small molecules and biologics with a focus on mAbs. He outlined the clearance mechanisms related to small molecules and biologics, described the $\mathrm{mAb}$ drug as a 'victim' (pharmacokinetics [PK] of mAbs impacted by other combinations of therapeutics) or as a perpetrator (where mAbs influence the PK of small molecules in a combination setting). A case study example of tocilizumab's DDI on the PK of simvastatin, and its clinical relevance, was discussed. Challenges with exploratory DDI studies and potential for false-positive DDIs due to suboptimal study designs were illustrated with a case study example of bevacizumab. Joleen White (Bristol-Myers Squibb) addressed the topic of identifying and overcoming antibody interference in ligand-binding PK assays. She outlined the challenges with interference in ligand-binding PK assays due to immunogenicity. Potential considerations for mitigation strategies in assay design, interference characterization and understanding interference in study samples were discussed. Three hypothetical case study examples were presented to illustrate the risk of antibody interference in PK assays and possible clinical interpretations. It is important to design assays to fit the purpose and characterize interferences to enable proper interpretation of analytical data.

The Thursday plenary session focused on method development and validation. Timothy P Foley (Genzyme) provided an overview of design of experiments (DOE) and why DOE is more effective for mapping the response surface than optimizing one factor at a time. The talk included the workflow for bioanalytical assay DOE and how the analytical factors are translated into statistical responses through estimation of model parameters using commercial software packages, and concluded with two examples of DOE in the bioanalytical laboratory: use of a d-optimal design for troubleshooting a ligand-binding assay; and use of a completely replicated fractional factorial design for screening potential interfering substances in a clinical 
LC-MS/MS assay. KC Van Horne's (Tandem Labs) talk focused on standardized paradigms for small-molecule LC-MS/MS method development. Case studies illustrated that the standardized approach can possibly shorten the duration of typical method development time from 2 weeks to 3 days. Under this paradigm, scientists use standard documentation where stock solutions are prepared using standardized solvents, and analyte stability and stickiness are routinely studied. The LC-MS/MS method development starts from a few commonly used LC columns, and phospholipids are always monitored. Chris Beaver from Montreal, Canada, discussed the challenges in the validation and use of biomarker kits. These kits are typically not made for drug development, their validation criteria are not stated; and they are designed for 'best matrix' situations for selectivity; but the benefits of these kits include being readily available, ease of use, low cost and easy transfer. Several examples were given for assay selectivity evaluation and remediation of selectivity-related issues. He used a case study to illustrate the value of monitoring quality control (QC) performance to support sample analysis for long-term studies to control QC issues with the kit components. The session concluded with an update on the bioanalytical method guidance. Brian Booth (US FDA) outlined the projected timelines for updating FDA bioanalytical guidance. He emphasized that the revised guidance will be largely based on the published white papers that have been discussed in the recent years [1,2]. There will not be any surprises in the updated FDA bioanalysis method guidance. CT Viswanathan (FDA) discussed new issues related to bioanalysis, such as microsampling/dried blood spot sampling, handling background interference issues for endogenous compounds and adapting of diagnostic kits. He voiced his strong support for harmonizing bioanalytical guidance across countries and regions. Mark E Arnold (BristolMyers Squibb) gave a brief update on the Global Bioanalysis Consortium (GBC), a newly formed group aiming at working with different groups and parts of the world to develop a consensus global bioanalytical guidance to present to health authorities.

The Friday morning plenary session featured two talks on laboratory frustrations. Eric David (Biogen Idec) described the efforts of the past 2 years in creating, staffing, training and starting work in a new laboratory to handle regulated preclinical and clinical sample analysis for both small molecules by LC-MS/MS and biologics by ligand-binding assays (ELISA). $\mathrm{He}$ presented some of the subtle differences between the LC-MS/MS and ELISA assay practices and described the activities, through the use of buddies, to crosstrain staff between LC-MS/MS and ELISA assays and technology. Utilizing this model they were able to develop better practices, standard operating procedures and reports templates as input is derived from both small- and large-molecule science, and move beyond good laboratory practice/regulated bioanalysis and seek clinical laboratory improvement amendments certification for performing biomarker analysis in clinical studies. Scott Vincent (Pfizer) then presented a summary of the challenges related to shipping early clinical samples from clinical sites to laboratories, when the laboratory locations are globally disperate. Planning, execution, management and documentation related to ensuring the successful delivery of samples stored in proper containers, at the proper temperature, on routine and critical timelines were discussed. The myth that samples could not ship over the weekend was dispelled. Success was also gained through partnerships with the company responsible for transporting the samples, as well as utilizing all of the technology that is available. A number of examples were provided on obtaining the correct speed of shipping to offset the cost of overnight shipments, including the status of current temperature loggers that can now go beyond temperature to box orientation and even GPS locating.

\section{Evening sessions}

The Tuesday evening session was on electronic laboratory notebooks (ELNs). Chad Briscoe (PRA International) reviewed some of the historical methods of bioanalytical data recording and noted that the bioanalytical community has reached a crossroads with respect to implementation of ELN systems. He described some of the inherent advantages associated with ELNs including improved quality, efficiency, record keeping, intellectual property protection, data sharing and documentation. He reviewed the various types of ELN architecture currently available including open-form systems, controlled forms interfaced with an Oracle database, Excel-based templates, Windows folders and various document management systems. For bioanalytical applications, desirable features of ELNs include the use of flexible forms for input of calibration information, material registration and reagent preparation. They should include templates with 'pick lists' for common items and be readily integrated with current 
laboratory instrumentation, existing laboratory information management systems and internal installation quantification and operational qualification or validation protocols. He then proceeded to describe his efforts with selection of a suitable ELN system for implementation within his organization. Some of the key parameters included the flexibility of the input formats and templates, the ease of integration with Watson laboratory information management system (LIMS) or instrument acquisition software, and the relative ease of implementation in a bioanalytical environment. His presentation included a summary of a brief online survey of conference attendees and noted that only $14 \%$ currently use ELNs in their operations. To provide additional information related to ELNs for attendees, a number of ELN vendors were asked to exhibit their ELN products following the presentation and during the Wednesday poster session (Waters Vision Publisher, IDBS BioBook, Lab Data Solutions Labnotes, Agilent ELN, Artus Ensemble ELN, and Cambridgesoft E-notebook). The vendor displays were directed at bioanalytical issues and provided a unique side-byside comparison of products.

The analytical investigators forum was held on Thursday evening and provided a platform for junior scientists to present research. Sarah Roby (Celerion) discussed the successes and challenges associated with the high-throughput analysis of large molecules. Large molecule analysis can be challenging because of the nature of the assays, which can involve varying incubation times, duplicate or triplicate analysis with dilution, and batch size limitations dependent on assay timing. The recommended approach for establishing a high-throughput method is to review the individual assay with respect to sample preparation/aliquoting, incubation steps and timing, equipment needs and solution preparation in order to identify the rate-limiting steps. Examples were given for optimization of assays for IGF-1 and IGFBP-3, PGE-2, PTH 1-34 and glucagon. Jun Zhang (Abbott Laboratories) presented the results of a case study on the conduct of an incurred sample reanalysis (ISR) investigation. He reviewed the recently published acceptance criteria for ISR, the approach to be taken for investigation of a failed ISR and discussed the case study. In this case, plasma samples were assayed for the drug and one metabolite using a fully validated method, and all runs were acceptable with no freeze-thaw or longterm stability issues. The investigation showed that other metabolites present in the incurred plasma samples could interfere, especially at the later sampling times (elimination phase), where parent drug concentrations were low. The method was modified (chromatography) to separate the interfering metabolites. It illustrates the importance of a thorough, well-documented investigation of ISR failure to identify the cause and method modification to insure reproducible performance. Sophie Pan (NoAb BioDiscoveries) discussed the utilization of solid phase microextraction (SPME) for the sampling and processing of in vivo samples. This technique integrates sampling, extraction, concentration and sample introduction for analysis into a single step. The method involves solvent-less extraction via a fused silica or stainless steel fiber coated with a thinfilm polymer and allowed for direct sampling and extraction in circulating blood with no blood loss, and determines the unbound-drug concentrations. A potential limitation is that SPME depends on sampling at equilibrium, which may take a long time. A pre-equilibrium sampling strategy and kinetic calibration can be used to avoid this limitation. An example of the use of SPME in vivo sampling for determination of unbound concentrations of five agents was given.

\section{Workshops, tutorials \& round-table discussions}

Tuesday afternoon's workshop session focused on the discussion regarding tissue sample analysis. Min Chang (Biogen Idec) presented an overview of the use of drug candidate concentration data in discovery and development and a case study on optimization of the homogenization process for the analysis of a drug candidate in the rat brain. The method development is difficult due to the limited availability of analyte-containing tissue and the lack of a reference for recovery. It was demonstrated that an analog of the analyte added to the crushed brain tissue prior to homogenizing correlated with the analyte recovery and has the potential to serve as an internal standard. Stacy Ho (Genzyme) focused on the practical aspects of brain tissue homogenization and considerations for improving throughput in support of discovery programs. She discussed in detail the impact of sample size on the homogenization time, solvents and tube when homogenizing mouse, rat and mouse neonatal brains. The results of drug exposures and biomarker reductions in plasma and brain were presented. Soma Ray (Biogen Idec) presented a case study involving measurement of a mAb drug concentration in the CNS to identify a suitable animal model of toxicology assessment. Ray discussed the challenges of measuring large-molecule therapeutics in tissues, and went on to describe processing of the brain and spinal 
cord samples using the Covaris Cryoprep and S2 homogenizer. Sample analysis and results from the case study where different animal models of blood-brain barrier penetration were compared with normal rats. After the presentations, there was an interactive panel discussion among the audiences, speakers and moderator related to:

- Building up confidence in the drug concentrations in tissue from the measurement of tissue homogenate samples;

- Established stability for tissue sample analysis;

- Practical considerations for dividing tissues for multiple tests;

- Achieving good recovery of large molecules during extraction;

- Assay validation for tissue sample analysis.

The Thursday afternoon workshop considered problems commonly encountered in daily laboratory work. Questions were provided by the attendees during the first 2 days and eight themes were discussed:

- Tissue analysis

- Performing selectivity in method validation

- Carryover effects

- Instrument response

- Stability studies

- Pipette verification

- Ligand-binding assays

- Dried blood spots

Discussions were highly interactive and many attendees gained new understanding of experiments that could be added to their own laboratories.

On Wednesday afternoon, two concurrent tutorials were presented. The first, presented by Balaji Agoram (MedImmune), dealt with absorption, distribution, metabolism and excretion and the disposition of proteins. In an adjacent room, Philip R Oldfield (Charles River Laboratories) presented a tutorial on the fundamentals of immunoassay development and validation. In both tutorials, there were active exchanges between the faculty and learners.

On Wednesday evening, following the poster session, informal round tables were held on several topics. These discussions were on:

- Transition from small-molecule BA to biologics BA (led by Danlin Wu, Roche);

- Navigating discrepant guidances for bioanalytical method validation and metabolites in safety testing (led by Eric Fluhler, Pfizer);

- Bioanalytical challenges for sample collection (led by Stacy Ho, Genzyme);

- Factors influencing LC-MS/MS calibration curve regression models (led by Qin Ji, Bristol-Myers Squibb);

- Tissue sample analysis (led by Min Chang, Biogen Idec).

\section{Conclusion}

In comparison to larger international conventions and because of the setting, attendees have more time to meet each other, discuss their organizations, practices and the science. Evaluation results were very positive and a summary is presented on the conference website [101]. The 2011 program is currently being developed and will be posted on the University's website when finished.

\section{Financial \& competing interests disclosure}

The authors have no relevant affliations or financial involvement with any organization or entity with a financial interest in or financial conflict with the subject matter or materials discussed in the manuscript. This includes employment, consultancies, honoraria, stock ownership or options, expert testimony, grants or patents received or pending, or royalties.

No writing assistance was utilized in the production of this manuscript.

\section{Bibliography}

1 Viswanathan CT, Bansal S, Booth B et al. Workshop/conference report - quantitative bioanalytical methods validation and implementation: best practices for chromatographic and ligand binding assays. AAPS J. 9(1), E30-E42 (2007).
2 Fast D. Workshop report and follow-up - AAPS workshop on current topics in GLP bioanalysis: assay reproducibility for incurred samples - implications of Crystal City recommendations. AAPS J. 11(2), 238-241 (2009).

\section{- Website}

101 11th Annual Land O'Lakes Bioanalytical Conference. A new decade of bioanalysis of large and small molecules. http://ce.pharmacy.wisc.edu/ courseinfo/2010JulyLOL 